\title{
A new triterpene saponin from Fagonia schimperi
}

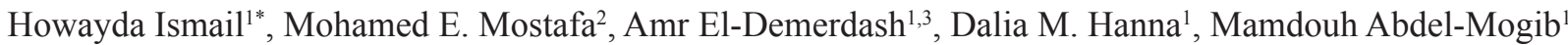 \\ ${ }^{1}$ Organic Chemistry Department, Faculty of Sciences, Mansoura University, Mansoura, Egypt. \\ ${ }^{2}$ Plant Protection Research Institute, Agriculture Research Center, Giza, Egypt. \\ ${ }^{3}$ Natural Products and Medicinal Chemistry Department, Institute de Chimie des Substances Naturelles, Gif-sur-Yvette, France.
}

\author{
ARTICLE INFO \\ Received on: 06/04/2020 \\ Accepted on: 14/09/2020 \\ Available online: 05/12/2020 \\ Key words: \\ Fagonia schimperi, \\ triterpene saponins fraction, \\ antibacterial, antioxidant, \\ cytotoxicity, 3-O- $\beta$ - \\ xylopyranosyl-( $(1 \rightarrow 2)-\alpha-$ \\ L-arabinopyranosyl-27- \\ hydroxyoleanolic acid \\ 28-O- $\beta$-glucopyranoside.ç
}

\begin{abstract}
Chemical investigation of the whole Fagonia schimperi C. Presl plant yielded a new triterpene saponin, namely 3-O- $\beta$ xylopyranosyl-(1 $\rightarrow 2)$ - $\alpha$-L-arabinopyranosyl-27-hydroxyoleanolic acid 28-O- $\beta$-glucopyranoside (1), along with five known natural products 2-6. Structures of the isolated compounds were elucidated by intensive spectroscopic methods including Electrospray Ionisation-Mass Spectrometry (ESI-MS) and 1D and 2D-NMR, which matched the literature data. Fagonia schimperi crude saponin fraction, ethyl acetate (EtOAc) fraction, and the new compound (1) were investigated for their biological activities as antibacterial, antioxidant, and cytotoxicity. EtOAc fraction and compound (1) were the most potent agents, wherein they displayed potent antibacterial activity against both Gram-positive (Staphylococcus aureus and Bacillus subtilis) and Gram-negative bacteria (Escherichia coli and Pseudomonas aeruginosa). Additionally, the EtOAc fraction displayed potent antiproliferative activity against hepatocellular carcinoma and mammary gland with $\mathrm{IC}_{50}$ values of $7.38 \pm 0.4$ and $4.89 \pm 0.3 \mu \mathrm{g} / \mathrm{ml}$, respectively. Furthermore, compound 1 showed a significant cytotoxic activity against the two cell lines with $\mathrm{IC}_{50}$ values of $19.20 \pm 1.4$ and $8.18 \pm 0.9, \mu \mathrm{g} / \mathrm{ml}$, respectively. This comes in agreement with the antioxidant activity that was assessed by 2,2,1-diphenyl-1-picrylhydrazyl and SOD-like activity methods in the following order: EtOAc fraction $>$ compound $\mathbf{1}>$ crude saponin fraction.
\end{abstract}

\section{INTRODUCTION}

Fagonia schimperi C. Presl belongs to the family Zygophyllaceae (Boulos, 2002). Historically, Fagonia species have been used in ancient medicines for the cure of jaundice, blood refinement, cold, cough, asthma, skin contagion, and liver issues and as carminative and emetic as well (Kanwal et al., 2017). The decoction of dried leaves or fresh juice of the whole plant is utilized in abdomen issues and fever (Kasture et al., 2014). Additionally, Fagonia species extracts are thought to exhibit numerous biopotentialities including antimicrobial, antiinflammatory, analgesic, and antipyretic activities (Nagaraj and Venkateswarlu, 2013). Many saponin compounds from different species of Fagonia have been used to treat many diseases, such as cancer and diabetes, in addition to having molluscicidal and

\footnotetext{
"Corresponding Author

Howayda Ismail, Organic Chemistry Department, Faculty of Sciences, Mansoura University, Mansoura, Egypt.

E-mail: howaydaismail15@gmail.com
}

antioxidant activities (Farheen et al., 2015; Lee et al., 2012; Saleem et al., 2014; Shaker et al., 2013). The meditative properties of this genus were coming from the diversity of its active phytochemical components. Previous phytochemical studies revealed the presence of a large number of triterpene glycosides, which are considered as major isolated secondary metabolites from Fagonia genus, in addition to sulfated triterpenoids, sulfated triterpenoid glycosides (Perrone et al., 2007; Shaker et al., 1999), diterpenes, sterols, flavonoids, proteins, amino acids, and coumarins, as well as trace elements (Abdel-Khalik et al., 2000; Abdel-Khalik et al., 2001; Ansari et al., 1987; El-Wakil, 2007; Shaker et al., 2013). Moreover, Fagonia cretica extract displayed potential anticancer activity against many forms of carcinoma by using it as herbal tea and a food additive, in addition to its use for the treatment of diabetes in the form of herbal tea (Lam et al., 2012; Nazir et al., 2017). Many Fagonia species have been chemically explored, and to the best of our knowledge, the literature survey indicated that F. schimperi C. Presl. is not investigated yet for its phytochemical constituents. Accordingly, and as a part of our research interest on pharmacologically active natural products (El-Demerdash et al., 
2009, 2012, 2018a, 2018b, 2019a, 2019b; El-Rokh et al., 2018), here we record the isolation, structural elucidation, and biological evaluations of a new triterpene saponin (1), along with five coisolated known compounds (2-6) from F. schimperi C. Presl. collected in Egypt (Fig. 1). It is worth mentioning that the present study is the first documentation centered on the phytochemical examination of F. schimperi C. Presl.

\section{EXPERIMENTAL}

\section{General}

NMR spectra were recorded on a Bruker 400 and Jeol $500 \mathrm{MHz}$ using tetramethylsilane as an internal standard. Deuterated solvents $\left(\mathrm{MeOH}-d_{4}\right.$, pyridine- $\left.d_{5}\right)$ were used for NMR measurements. Mestrenova software was used in processing the recorded 1 and 2D-NMR spectra, which were calibrated using ${ }^{1} \mathrm{H}$ and ${ }^{13} \mathrm{C}$ solvent signals for $\mathrm{MeOH}-d_{4}$ at $\delta_{\mathrm{H}} 3.31, \delta_{\mathrm{C}} 49.15$, and pyridine $-d_{5}$ at $\delta_{\mathrm{H}} 8.74,7.58,7.22, \delta_{\mathrm{C}} 150.35,135.91$, and 123.87 . The chemical shift $(\delta)$ values were given in ppm and the coupling constants $(J)$ in Hz. The acronyms s, br s, d, t, q, and dd are used for single, broad singlet, doublet, triplet, quartet, and doublet of doublet, Mansoura University, Egypt. Mass spectra were carried out on an API Q-STAR PULSAR I of applied biosystem by direct injection of the purified compounds, Central national de la recherché scientifique (CNRS), France. Silica gel (Kieselgel 60, F 254) of $0.25 \mathrm{~mm}$ thickness was used for carrying out thin-layer chromatography and preparative Thin Layer Chromatography (TLC). Column chromatography was carried out using silica gel F254 (230-400 mesh), Sephadex LH-20, and/or polyamide 6.

\section{Solvents}

The solvents acetone, hexane, dichloromethane (DCM), ethyl acetate (EtOAc), and methanol $(\mathrm{MeOH})$ were aquired from Adwic Company, Mansoura, Egypt.

\section{Biological materials}

Anticancer activity was carried out in vitro by utilizing two human cancer cell lines, hepatocellular carcinoma (HePG-2), and mammary gland (MCF-7), gained by US National Cancer Institute, according to the announced standard procedure qualified by Skehan et al. (1990). 2,2,1-diphenyl-1-picrylhydrazyl (DPPH) and L-ascorbic acids were acquired from Aldrich Chemical Co., USA, while pure EtOH (of analytical grade) was acquired from El-Nasr Co. for Pharmaceutical Chemicals, Egypt. This test was carried out in accordance with the procedure represented by Prouillac et al. (2009). The antibacterial activity was carried out by using the conventional broth dilution method (Rahman et al., 2001). Ampicillin was used as a reference drug.

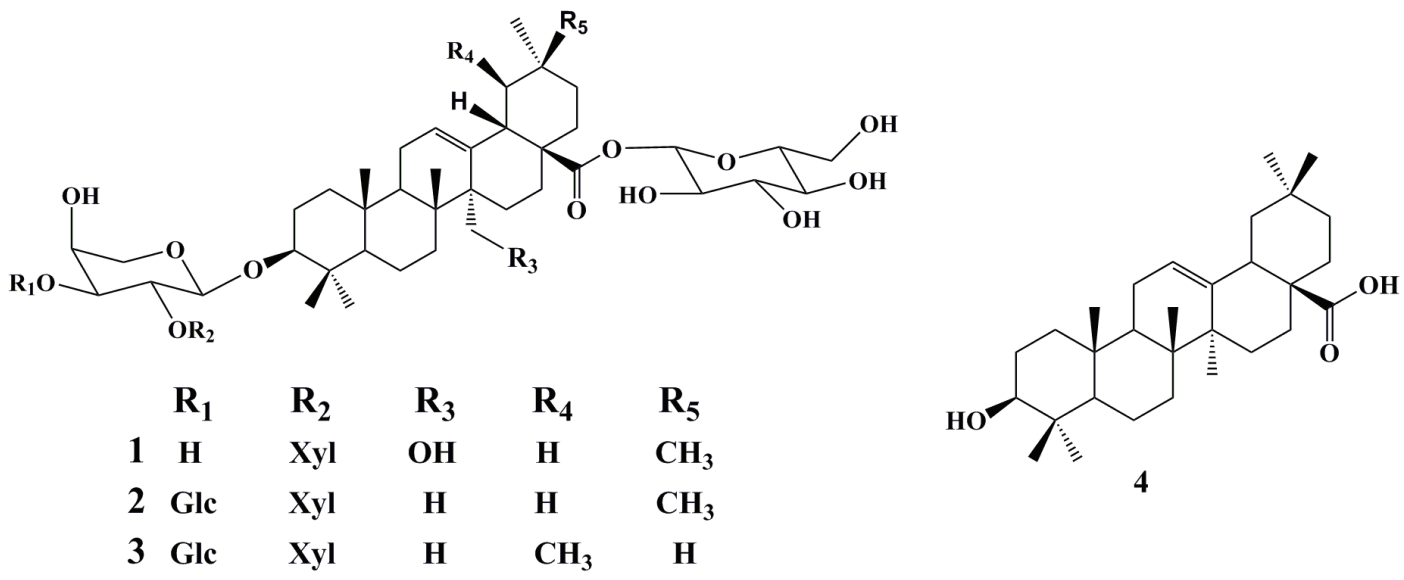<smiles>[R]c1cc(-c2oc3c(OC)c(O)cc(O)c3c(=O)c2OC(O)COC(OC2OC(C)C(O)C(O)C2O)C(O)C(O)C(O)O)ccc1O</smiles>

Figure 1. Isolated compounds 1-6 from F. schimperi C. Presl. 


\section{Plant material}

F. schimperi C. Presl was collected by the third author from Saint Catherine District, South Sinai Governorate, Egypt, in March 2010, and had been identified by Professor Loutfy Boulos, National Research Centre, Cairo, Egypt. A voucher specimen was deposited at Herbarium of Botany Department, Faculty of Science, Mansoura University.

\section{Processing of plant material}

The air-dried whole plant powder (250 g) was ground and exhaustively extracted with $\mathrm{MeOH}(3 \times 11)$ at room temperature. Then, the methanolic extract was collected and dried under reduced pressure up to one-third of its volume and further diluted with water. Successive liquid-liquid solvent partitioning was used, using different organic solvents starting with hexane (2L), DCM (2L), and finally EtOAc (2L). The organic extracts were combined and dried under reduced pressure to afford three fractions: hexane fraction $(6 \mathrm{~g}), \mathrm{DCM}$ fraction (1.5 g), and EtOAc fraction ( $3 \mathrm{~g})$. The remaining aqueous layer was subjected to amberlite ion exchanger and was preliminarily eluted with distilled water and then with $\mathrm{MeOH}$. The $\mathrm{MeOH}$ fraction was collected and evaporated under reduced pressure to give a brown residue $(40 \mathrm{~g})$. The residue was redissolved in the minimum amount of $\mathrm{MeOH}$ and saturated with cold acetone to precipitate the crude saponin fraction as brown gummy material $(10 \mathrm{~g})$.

The EtOAc fraction (3 g) was chromatographed on polyamide in six columns and eluted with $\mathrm{H}_{2} \mathrm{O} 100 \%, \mathrm{H}_{2} \mathrm{O}: \mathrm{MeOH}$ (1:1), $\mathrm{MeOH} 100 \%, \mathrm{MeOH}: \mathrm{Me}_{2} \mathrm{CO}$ (1:1), $\mathrm{Me}_{2} \mathrm{CO} 100 \%, \mathrm{Me}_{2} \mathrm{CO}$ : $\mathrm{NH}_{4} \mathrm{OH}(1: 1)$, and $\mathrm{NH}_{4} \mathrm{OH} 100 \%$, respectively. All fractions were monitored by TLC, where similar fractions were combined based on their TLC profiling, to afford 10 major subfractions. Subfraction (I) was further chromatographed on Sephadex LH-20, eluted with $\mathrm{MeOH}(100 \%)$, and then purified on preparative silica gel TLC plates using EtOAc:MeOH: $\mathrm{H}_{2} \mathrm{O}$ (14:3:1) as a developing system to give a pale yellow powder of compound 1 ( $\left.80 \mathrm{mg}, R_{\mathrm{f}} 0.54\right)$. Subfraction (II) was further separated over a silica gel column (DCM:MeOH) (40:1) and purified by PTLC silica gel plates (EtOAc:MeOH) (14:3) to give an amorphous powder of compound 4 (40 mg, $R_{\mathrm{f}}$ 0.31). Subfraction (III) was chromatographed on silica gel PTLC plates (EtOAc: $\left.\mathrm{MeOH}: \mathrm{H}_{2} \mathrm{O}\right)(13: 3: 1)$ to yield a yellow powder of compounds 5 (70 $\left.\mathrm{mg}, R_{\mathrm{f}} 0.43\right)$ and $\mathbf{6}\left(50 \mathrm{mg}, R_{\mathrm{f}}\right.$ $0.45)$. The crude saponins fraction was chromatographed on silica gel column with an isocratic system (EtOAc:DCM:MeOH:H $\mathrm{H}_{2} \mathrm{O}$ ) (6:4:4:1) to yield three main subfractions: (i) (3 g), (ii) (4 g), and (iii) (2 g). Fraction I was further chromatographed on Sephadex LH-20 (MeOH: $\mathrm{H}_{2} \mathrm{O}$ 17: 3) to yield a subfraction, which was purified on silica gel PTLC plates using EtOAc:MeOH: $\mathrm{H}_{2} \mathrm{O}$ (13:3:1) as a developing system to yield an amorphous powder of compounds 2 (70 mg, $R_{\mathrm{f}} 0.42$ ) and $\mathbf{3}$ (55 mg, $\left.R_{\mathrm{f}} 0.40\right)$.

\section{Spectral characterization of the isolated compounds}

3-O- $\beta$-Xylopyranosyl- $(1 \rightarrow 2)-\alpha$-L-arabinopyranosyl-27hydroxyoleanolic acid 28-O- $\beta$-glucopyranoside (1): pale yellow powder; Proton Nuclear Magnetic Resonance $\left({ }^{1} \mathrm{H}\right.$ NMR) and ${ }^{13} \mathrm{C}$ NMR (see Table 1); Electrospray Ionisation-Mass Spectrometry (ESI-MS) negative ion mode [M-H] ${ }^{-}$ion at $\mathrm{m} / \mathrm{z} 897$ and positive ion mode as sodium salt $[\mathrm{M}+\mathrm{Na}]^{+}$ion at $\mathrm{m} / z 921$ (calcd. for $\left.\mathrm{C}_{46} \mathrm{H}_{74} \mathrm{O}_{17}, 898\right)$.
Table 1. ${ }^{1} \mathrm{H}$ and ${ }^{13} \mathrm{C}$ NMR spectral data of compounds 1 in $\mathrm{MeOH}-d_{4}(500$ and $125 \mathrm{MHz})$.

\begin{tabular}{|c|c|c|}
\hline \multirow{2}{*}{ Position } & \multicolumn{2}{|c|}{1} \\
\hline & $\delta_{\mathrm{H}}$, multiplicity $(\mathrm{J}$ in $\mathrm{Hz})$ & $\delta_{\mathrm{C}}$, Type \\
\hline 1 & $0.99,1.58, m$ & $39.67, \mathrm{CH}_{2}$ \\
\hline 2 & $1.69,1.83, m$ & $27.07, \mathrm{CH}_{2}$ \\
\hline 3 & $3.11, d d(4.6,11.5)$ & $90.88, \mathrm{CH}$ \\
\hline 4 & - & $40.29, \mathrm{Cq}$ \\
\hline 5 & $0.85, m$ & $56.81, \mathrm{CH}$ \\
\hline 6 & $1.37,1.53, m$ & $19.31, \mathrm{CH}_{2}$ \\
\hline 7 & $1.62,1.31, m$ & $34.27, \mathrm{CH}_{2}$ \\
\hline 8 & - & $41.30, \mathrm{Cq}$ \\
\hline 9 & $1.86, m$ & $49.53, \mathrm{CH}$ \\
\hline 10 & - & $38.02, \mathrm{Cq}$ \\
\hline 11 & $1.41,1.88, m$ & $24.92, \mathrm{CH}_{2}$ \\
\hline 12 & $5.62, t(3.2)$ & 129.15, CH \\
\hline 13 & - & $139.47, \mathrm{Cq}$ \\
\hline 14 & - & $48.49, \mathrm{Cq}$ \\
\hline 15 & $1.71,1.31, m$ & $23.72, \mathrm{CH}_{2}$ \\
\hline 16 & $2.19,1.71, m$ & $24.33, \mathrm{CH}_{2}$ \\
\hline 17 & - & $47.77, \mathrm{Cq}$ \\
\hline 18 & $2.92, d d(3.4,13.0)$ & $42.30, \mathrm{CH}$ \\
\hline 19 & $1.20,1.56, m$ & $46.13, \mathrm{CH}_{2}$ \\
\hline 20 & - & $31.55, \mathrm{Cq}$ \\
\hline 21 & $1.22,1.41, m$ & $34.71, \mathrm{CH}_{2}$ \\
\hline 22 & $1.58,1.73, m$ & $33.12, \mathrm{CH}_{2}$ \\
\hline 23 & $1.03, s$ & $28.27, \mathrm{CH}_{3}$ \\
\hline 24 & $0.82, s$ & $16.60, \mathrm{CH}_{3}$ \\
\hline 25 & $0.93, s$ & $16.40, \mathrm{CH}_{3}$ \\
\hline 26 & $0.75, s$ & $19.04, \mathrm{CH}_{3}$ \\
\hline 27 & $3.46 / 3.73, \mathrm{~m}$ & $64.88, \mathrm{CH}_{2}$ \\
\hline 28 & - & $178.07, \mathrm{Cq}$ \\
\hline 29 & $0.91, s$ & $33.39, \mathrm{CH}_{3}$ \\
\hline 30 & $0.95, s$ & $24.05, \mathrm{CH}_{3}$ \\
\hline \multicolumn{3}{|l|}{ Ara } \\
\hline $1^{\prime}$ & $4.44, d(6.9)$ & $105.41, \mathrm{CH}$ \\
\hline $2^{\prime}$ & 3.78 & $80.53, \mathrm{CH}$ \\
\hline $3^{\prime}$ & 3.74 & $73.53, \mathrm{CH}$ \\
\hline $4^{\prime}$ & 3.84 & $68.86, \mathrm{CH}$ \\
\hline $5^{\prime}$ & $3.50,3.83, m$ & $65.28, \mathrm{CH}_{2}$ \\
\hline \multicolumn{3}{|l|}{ Xyl } \\
\hline $1^{\prime \prime}$ & $4.46, d(7.6)$ & $106.13, \mathrm{CH}$ \\
\hline $2^{\prime \prime}$ & 3.21 & $75.88, \mathrm{CH}$ \\
\hline $3^{\prime \prime}$ & 3.32 & $77.79, \mathrm{CH}$ \\
\hline $4^{\prime \prime}$ & $3.47, d d(10.1,10.3)$ & $71.12, \mathrm{CH}$ \\
\hline $5^{\prime \prime}$ & $3.16 / 3.81, m$ & $67.11, \mathrm{CH}_{2}$ \\
\hline \multicolumn{3}{|l|}{ 28-GLC } \\
\hline $1^{\prime \prime \prime}$ & $5.39, d(8.01)$ & $95.78, \mathrm{CH}$ \\
\hline $2^{\prime \prime \prime}$ & 3.33 & 73.91, CH \\
\hline $3^{\prime \prime \prime}$ & 3.36 & $78.69, \mathrm{CH}$ \\
\hline $4^{\prime \prime \prime}$ & 3.35 & $71.15, \mathrm{CH}$ \\
\hline $5^{\prime \prime \prime}$ & 3.41 & $78.28, \mathrm{CH}$ \\
\hline $6^{\prime \prime \prime}$ & $3.68,3.82, m$ & $62.41, \mathrm{CH}_{2}$ \\
\hline
\end{tabular}


3 - O - $\beta$ - D - X y lo p y r a n o s y $1(1 \rightarrow 2)-[\beta-\mathrm{D}-$ glucopyranosy $(1 \rightarrow 3)]$ - $\alpha$-arabinopyranosyloleanolic acid $28-\mathrm{O}-\beta$ glucopyranoside (2): amorphous powder; ESI-MS negative ion mode $[\mathrm{M}+\mathrm{HCOOH}-\mathrm{H}]^{-}$ion at $m / z \quad 1,089$ and positive ion mode $[\mathrm{M}+\mathrm{H}]^{+}$ion at $m / z 1,045$ (calcd. for $\mathrm{C}_{52} \mathrm{H}_{84} \mathrm{O}_{21}, 1,044$ ). ${ }^{1} \mathrm{H}$ and ${ }^{13} \mathrm{C}$ NMR $\left(500,125 \mathrm{MHz}, \mathrm{MeOH}-d_{4}\right): 3.10(1 \mathrm{H}, \mathrm{m}, \mathrm{H}-3,91.29), 5.19$ $(1 \mathrm{H}$, brs, H-12, 123.95), $2.85(1 \mathrm{H}, \mathrm{dd}, J=2.84,9.16 \mathrm{~Hz}, \mathrm{H}-18$, 42.74), 1.05 (3H, s, H-23, 28.43), 0.83 (3H, s, H-24, 16.87), 0.95 $(3 \mathrm{H}, \mathrm{s}, \mathrm{H}-25,16.18), 0.80$ (3H, s, H-26, 17.82), 1.15 (3H, s, H-27, 26.50), 0.91 (3H, s, H-29, 33.66), 0.93 (3H, s, H-30, 24.20), 4.33 $(1 \mathrm{H}, \mathrm{d}, J=6.7 \mathrm{~Hz}$, Ara. H-1, 106.16), 3.84 (1H, m, Ara. H-2, 77.71), 3.83 (1H, m, Ara. H-3, 84.18), 4.02 (1H, s, Ara. H-4, 69.76), 3.78/3.55 (2H, m, Ara. H-5, 66.40), 4.55 (1H, d, $J=7.70$ Hz, Xyl. H-1, 105.14), 3.12 (1H, m, Xyl. H-2, 76.06), 3.35 (1H, m, Xyl. H-3, 78.47), 3.42 (1H, m, Xyl. H-4, 71.71), 3.77/3.14 (2H, m, Xyl. H-5, 67.01), 4.63 (1H, d, $J=7.75$ Hz, Glc. H-1, 105.01), 3.32 (1H, m, Glc. H-2, 75.63), 3.34 (1H, m, Glc. H-3, 78.77), $3.30(1 \mathrm{H}$, m, Glc. H-4, 71.30), 3.27 (1H, m, Glc. H-5, 78.02), 3.82/3.68 (2H, m, Glc. H-6, 62.49), 5.32 (1H, d, $J=6.65$, Glc. ester. H-1, 95.75), 3.31 (1H, m, Glc. ester. H-2, 73.64), 3.39 (1H, m, Glc. ester. H-3, 78.20), 3.34 (1H, m, Glc. ester. H-4, 71.23 ), 3.37 (1H, m, Glc. ester. H-5, 78.37), and 3.82/3.68 (2H, m, Glc. ester. H-6, 62.49), previously reported by Miyase et al. (1996).

3 - O - $\beta$ - D - X y l o p y ra no s y $1(1 \rightarrow 2)$ - $[\beta-\mathrm{D}-$ glucopyranosyl $(1 \rightarrow 3)]$ - $\alpha$-L-arabinopyranosylurslolic acid 28-O- $\beta$-glucopyranoside (3): amorphous powder; ESIMS negative ion mode [M+HCOOH-H] ion at $\mathrm{m} / z \quad 1,089$ and positive ion mode $[\mathrm{M}+\mathrm{H}]^{+}$ion at $\mathrm{m} / z$ 1,045 (calcd. for $\left.\mathrm{C}_{52} \mathrm{H}_{84} \mathrm{O}_{21}, 1,044\right) .{ }^{1} \mathrm{H}$ and ${ }^{13} \mathrm{C}$ NMR $(500,125 \mathrm{MHz}, \mathrm{MeOH})$ : 3.10 (1H, m, H-3, 91.29), 5.19 (1H, brs, H-12, 123.95), 2.20 $(1 \mathrm{H}, \mathrm{d}, J=9 \mathrm{~Hz}, \mathrm{H}-18,54.32), 1.05$ (3H, s, H-23, 28.43), 0.84 $(3 \mathrm{H}, \mathrm{s}, \mathrm{H}-24,16.87), 0.95$ (3H, s, H-25, 16.18), 0.83 (3H, s, H-26, 18.06), 1.11 (3H, s, H-27, 24.12), 0.89 (3H, d, $J=4.28$ Hz, H-29, 17.89), 0.96 (3H, d, $J=3.1 \mathrm{~Hz}, \mathrm{H}-30,21.71), 4.33$ $(1 \mathrm{H}, \mathrm{d}, J=6.7 \mathrm{~Hz}$, Ara. H-1, 106.16), 3.84 (1H, m, Ara. H-2, 77.71), 3.83 (1H, m, Ara. H-3, 84.18), 4.02 (1H, s, Ara. H-4, 69.76), 3.78/3.55 (2H, m, Ara. H-5, 66.40), $4.55(1 \mathrm{H}, \mathrm{d}, J=$ $7.70 \mathrm{~Hz}$, Xyl. H-1, 105.14), 3.12 (1H, m, Xyl. H-2, 76.06), 3.35 (1H, m, Xyl. H-3, 78.47), 3.42 (1H, m, Xyl. H-4, 71.71), $3.77 / 3.14(2 \mathrm{H}, \mathrm{m}, \mathrm{Xyl} . \mathrm{H}-5,67.01), 4.63(1 \mathrm{H}, \mathrm{d}, J=7.75 \mathrm{~Hz}$, Glc. H-1, 105.01), 3.32 (1H, m, Glc. H-2, 75.63$), 3.34$ (1H, m, Glc. H-3, 78.77), 3.30 (1H, m, Glc. H-4, 71.30), $3.27(1 \mathrm{H}, \mathrm{m}$, Glc. H-5, 78.02), 3.82/3.68 (2H, m, Glc. H-6, 62.49), $5.32(1 \mathrm{H}$, $\mathrm{d}, J=6.65$, Glc. ester. H-1, 95.75), 3.31(1H, m, Glc. ester. H-2, 73.64), 3.39 (1H, m, Glc. ester. H-3, 78.20$), 3.34$ (1H, m, Glc. ester. H-4, 71.23), 3.37 (1H, m, Glc. ester. H-5, 78.37), and 3.82/3.68 (2H, m, Glc. ester. H-6, 62.49), previously reported by Miyase et al. (1996).

Oleanolic acid (4): its molecular formula, $\mathrm{C}_{30} \mathrm{H}_{50} \mathrm{O}_{2}$, was determined on the basis of EI-MS; it gave $\mathrm{M}^{+}$at $m / z$ 456. ${ }^{1} \mathrm{H}$ NMR (500 MHz, pyridine- $\left.d_{5}\right): 3.44(1 \mathrm{H}, \mathrm{dd}, J=5.85,10.4 \mathrm{~Hz}, \mathrm{H}-3)$, 5.49 (1H, brs, H-12), 3.30 (1H, dd, $J=4.02,13.85 \mathrm{~Hz}, \mathrm{H}-18)$, 1.23 (3H, s, H-23), 1.02 (3H, s, H-24), 0.88 (3H, s, H-25), 1.00 (3H, s, H-26), 1.27 (3H, s, H-27), 0.94 (3H, s, H-29), and 1.02 (3H, s, H-30), in accordance with previously reported data by Rizk et al. (1972).
Herbacetin 8-methyl ether 3-rutinoside (5): ESI-MS positive ion mode as sodium salt $[\mathrm{M}+\mathrm{Na}]^{+}$ion at $\mathrm{m} / z 647$ (calcd. for $\left.\mathrm{C}_{28} \mathrm{H}_{32} \mathrm{O}_{16}, 624\right) .{ }^{1} \mathrm{H}$ and ${ }^{13} \mathrm{C}$ NMR $\left(500,125 \mathrm{MHz}, \mathrm{MeOH}-d_{4}\right)$ : 6.28 (1H, s, H-6, 100.31), 8.13 (1H, d, $\left.J=6 \mathrm{~Hz}, \mathrm{H}-2^{\prime}, 6^{\prime}, 132.52\right)$, $6.91\left(1 \mathrm{H}, \mathrm{d}, J=6 \mathrm{~Hz}, \mathrm{H}-3^{\prime}, 5^{\prime}\right), 3.90\left(3 \mathrm{H}, \mathrm{s}, 8-\mathrm{OCH}_{3}, 62.22\right), 5.16$ (1H, d, $\left.J=5 \mathrm{~Hz}, \mathrm{H}-1^{\prime \prime}, 104.69\right), 3.44$ (1H, m, H-2", 75.09), 3.33 (1H, m, H-3", 77.41), 3.28 (1H, m, H-4", 71.64), 3.42 (1H, m, H-5", 78.28), 3.82/3.41 (2H, m, H-6", 68.77), 4.51 (1H, d, $J=1.04$ Hz, H-1"', 102.60), 3.61 (1H, dd, $J=1.08,2.28$ Hz, H-2"', 72.20), 3.51 (1H, dd, $\left.J=2.24,6.36 \mathrm{~Hz}, \mathrm{H}-3^{\prime \prime \prime}, 72.26\right), 3.31$ (1H, m, H-4"', $74.01), 3.43$ (1H, m, H-5"', 69.89), and $1.11(3 \mathrm{H}, \mathrm{d}, J=4.12 \mathrm{~Hz}$, $\left.\mathrm{CH}_{3}-6^{\prime \prime \prime} 18.04\right)$, in accordance with previously reported data by Saleh and El-Hadidi (1977).

Limocitrin 3-O-rutinoside (6): ESI-MS positive ion mode as sodium salt $[\mathrm{M}+\mathrm{Na}]^{+}$ion at $m / z 677$ (calcd. for $\mathrm{C}_{29} \mathrm{H}_{34} \mathrm{O}_{17}$, 654). ${ }^{1} \mathrm{H}$ and ${ }^{13} \mathrm{C}$ NMR $\left(500,125 \mathrm{MHz}, \mathrm{MeOH}-d_{4}\right): 6.28(1 \mathrm{H}, \mathrm{s}$, $\mathrm{H}-6,100.29), 8.00$ (1H, d, $\left.J=1.4 \mathrm{~Hz}, \mathrm{H}-2^{\prime}, 114.49\right), 6.94$ (1H, d, $\left.J=5.6 \mathrm{~Hz}, \mathrm{H}-5^{\prime}, 116.34\right), 7.74\left(1 \mathrm{H}, \mathrm{dd}, J=1.4,5.6 \mathrm{~Hz}, \mathrm{H}-6^{\prime}\right.$, 124.22), $3.92\left(3 \mathrm{H}, \mathrm{s}, 8-\mathrm{OCH}_{3} 62.22\right), 3.96\left(3 \mathrm{H}, \mathrm{s},{ }^{3} 3-\mathrm{OCH}_{3} 56.85\right)$, $5.26\left(1 \mathrm{H}, \mathrm{d}, J=5.08 \mathrm{~Hz}, \mathrm{H}-1^{\prime \prime}, 104.48\right), 3.43(1 \mathrm{H}, m, \mathrm{H}-2 ", 76.06$, $3.31(1 \mathrm{H}, \mathrm{m}, \mathrm{H}-3 ", 77.55), 3.26(1 \mathrm{H}, \mathrm{m}, \mathrm{H}-4 ", 71.81), 3.40(1 \mathrm{H}$, m, H-5", 78.31), 3.80/3.42 (2H, m, H-6", 68.75), 4.52 (1H, d, J $\left.=1.04 \mathrm{~Hz}, \mathrm{H}-1^{\prime \prime \prime}, 102.70\right), 3.60$ (1H, dd, $J=1.08,2.24 \mathrm{~Hz}, \mathrm{H}-2^{\prime \prime \prime}$, 72.24), 3.47 (1H, dd, $\left.J=2.24,5.04 \mathrm{~Hz}, \mathrm{H}-3^{\prime \prime \prime}, 72.44\right), 3.25(1 \mathrm{H}$, m, H-4"', 73.94), 3.39 (1H, m, H-5"', 69.94), and 1.11 (3H, d, $J=$ $\left.4.16 \mathrm{~Hz}, 6^{\prime \prime \prime} \mathrm{CH}_{3} 18.03\right)$, in accordance with previously reported data by Ito et al. (2000).

\section{RESULTS AND DISCUSSION}

\section{Phytochemical evaluation}

A new triterpene saponin (1) has been identified from the EtOAc fraction, where its structure was resolved based on a combination of spectral data analyses, including ESIMS and 1D and 2D-NMR. Additionally, other five known coisolated compounds 2-6 were isolated and identified as 3 -O- $\beta$-D-xylopyranosyl $(1 \rightarrow 2)$-[ $\beta$-D-glucopyranosy $(1 \rightarrow 3)]$ $\alpha$-L-arabinopyranosyloleanolic acid 28-O- $\beta$-glucopyranoside (2) (Miyase et al., 1996), 3-O- $\beta$-D-xylopyranosyl( $1 \rightarrow 2)-[\beta$ D-glucopyranosyl $(1 \rightarrow 3)]$ - $\alpha$-L-arabinopyranosylurslolic acid

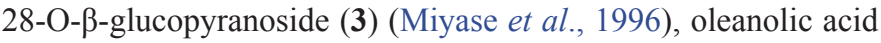
(4) (Rizk et al., 1972), herbacetin 8-methyl ether 3-rutinoside (5) (Saleh and El-Hadidi, 1977), and limocitrin 3-O-rutinoside (6) (Ito et al., 2000) by comparing their ${ }^{1} \mathrm{H}$ NMR spectral data with those previously reported in the literature. However, these compounds are reported for the first time from the species under investigation (Fig. 1).

Compound $\mathbf{1}$ was isolated as pale yellow powder. The molecular formula was assigned from its negative and positive ESI mass spectra as $\mathrm{C}_{46} \mathrm{H}_{74} \mathrm{O}_{17}$, as determined from [M-H] ion at $m / z 897$ and $[\mathrm{M}+\mathrm{Na}]^{+}$ion at $\mathrm{m} / \mathrm{z}$ 921. The ${ }^{1} \mathrm{H}$ NMR spectrum (Table 1) showed a triterpenoid pattern with singlets of six upfield tertiary methyl groups at $\delta_{\mathrm{H}} 0.75,0.82$, $0.91,0.93,0.95$, and $1.03 \mathrm{ppm}$, with a characteristic $\mathrm{H}-3$ signal at $\delta_{\mathrm{H}} 3.11(1 \mathrm{H}, \mathrm{dd}, J=4.6,11.5 \mathrm{~Hz})$, assigned to a $\mathrm{C}-3-\beta-\mathrm{OH}$ functionality. Furthermore, the characteristic endocyclic olefinic 
signal of oleanolic acid, $\mathrm{H}-12$, assigned at $\delta_{\mathrm{H}} 5.62(\mathrm{t}, J=3.2$ $\mathrm{Hz}$ ), with a downfield shift in agreement with the presence of a $\mathrm{CH}_{2} \mathrm{OH}$ group at $\mathrm{C}-14\left(\delta_{\mathrm{H}} 3.46\right.$ and $\left.3.73 \mathrm{ppm}\right)$ confirms that compound 1 features a _amyrin pentacyclic triterpene scaffold. The ${ }^{13} \mathrm{C}$ NMR along with ${ }^{1} \mathrm{H}-{ }^{13} \mathrm{C}$ Heteronuclear Single Quantum Coherence (HSQC) spectral data confirmed the presence of 46 carbon resonances corresponding to a 27 -hydroxy oleanolic acid and three sugar residues (Shaker et al., 2000). Extensive NMR data interpretations including $\mathrm{H}^{1}-\mathrm{H}^{1}$ COSY, ${ }^{13} \mathrm{C}$ NMR, and ${ }^{1} \mathrm{H}-{ }^{13} \mathrm{C}$ HSQC spectra allowed the full assignment of chemical shift values of the pentacyclic core and each sugar residue and showed the presence of $\beta$-glucopyranosyl, $\alpha$-Larabinopyranosyl, and $\beta$-xylopyranosyl moieties as illustrated in Table 1 (Shaker et al., 2000). The positions of attachment of the sugar units together with the connection sequences of each sugar moiety and the location of the $-\mathrm{CH}_{2} \mathrm{OH}$ group at $\mathrm{C}-14$ of the aglycone core were assigned by ${ }^{1} \mathrm{H}-{ }^{13} \mathrm{C}$ Heteronuclear Multiple Bond Correlation (HMBC) spectrum (Fig. 2). Significant HMBC correlations were observed between $\mathrm{H}-1$ (Glc ester) $\rightarrow \mathrm{C}-28$ and $\mathrm{H}-1$ (Ara) $\rightarrow \mathrm{C}-3$, which indicated the glycosidic linkage positions of $\beta$-glucopyranosyl and $\alpha$-L-arabinopyranosyl moieties to the aglycone. Further key correlations were observed between H-1 $(\mathrm{Xyl}) \rightarrow \mathrm{C}-2$ (Ara), which confirms the interglycosidic linkage between the terminal $\beta$-xylopyranosyl and $\alpha$-L-arabinopyranosyl moieties. Additional key HMBC correlation was observed from the anomeric proton of _-D-glucopyranose moiety $\left(\delta_{\mathrm{C}} 5.39\right.$, d, $8.01 \mathrm{~Hz})$ to the quaternary carbon $\left(\delta_{\mathrm{C}} 178.07, \mathrm{Cq}-28\right)$, which confirms the linkage to an ester functionality. The relative configurations of the anomeric protons were deduced by the ${ }^{3} J_{\mathrm{H} 1-\mathrm{H} 2}$ coupling constants analysis and comparing their ${ }^{13} \mathrm{C}-\mathrm{NMR}$ with those reported in the literature (Table 1). Thus, the structure of compound 1 was identified as 3-O- $\beta$-xylopyranosyl- $(1 \rightarrow 2)$ $\alpha$-L-arabinopyranosyl-27-hydroxyoleanolic acid 28-O- $\beta$ glucopyranoside.

\section{Biological activities assessment}

Almost $1 \mathrm{mg}$ of EtOAc, crude saponins fractions, and compound 1 was dissolved in Dimethyl sulfoxide (DMSO) and submitted for biological assays, including antibacterial, antioxidant, and cytotoxic activities.

\section{Antimicrobial activity assessment}

The antimicrobial activity of compound $\mathbf{1}$ and those of EtOAc and crude saponins fraction were compared with the activity of ampicillin and are presented in Table 2. Compound $\mathbf{1}$, EtOAc, and crude saponins exhibited remarkable activity against a panel of bacterial strains. It was observed that EtOAc fraction and compound $\mathbf{1}$ exhibited the highest activity against Gram-positive bacteria (Staphylococcus aureus and Bacillus subtilis) and Gramnegative bacteria (Escherichia coli and Pseudomonas aeruginosa). Crude saponin had moderate activity toward Bacillus subtilis (Gram-positive bacterium) and low activity toward Escherichia coli (Gram-negative bacterium).

\section{Cytotoxic activity}

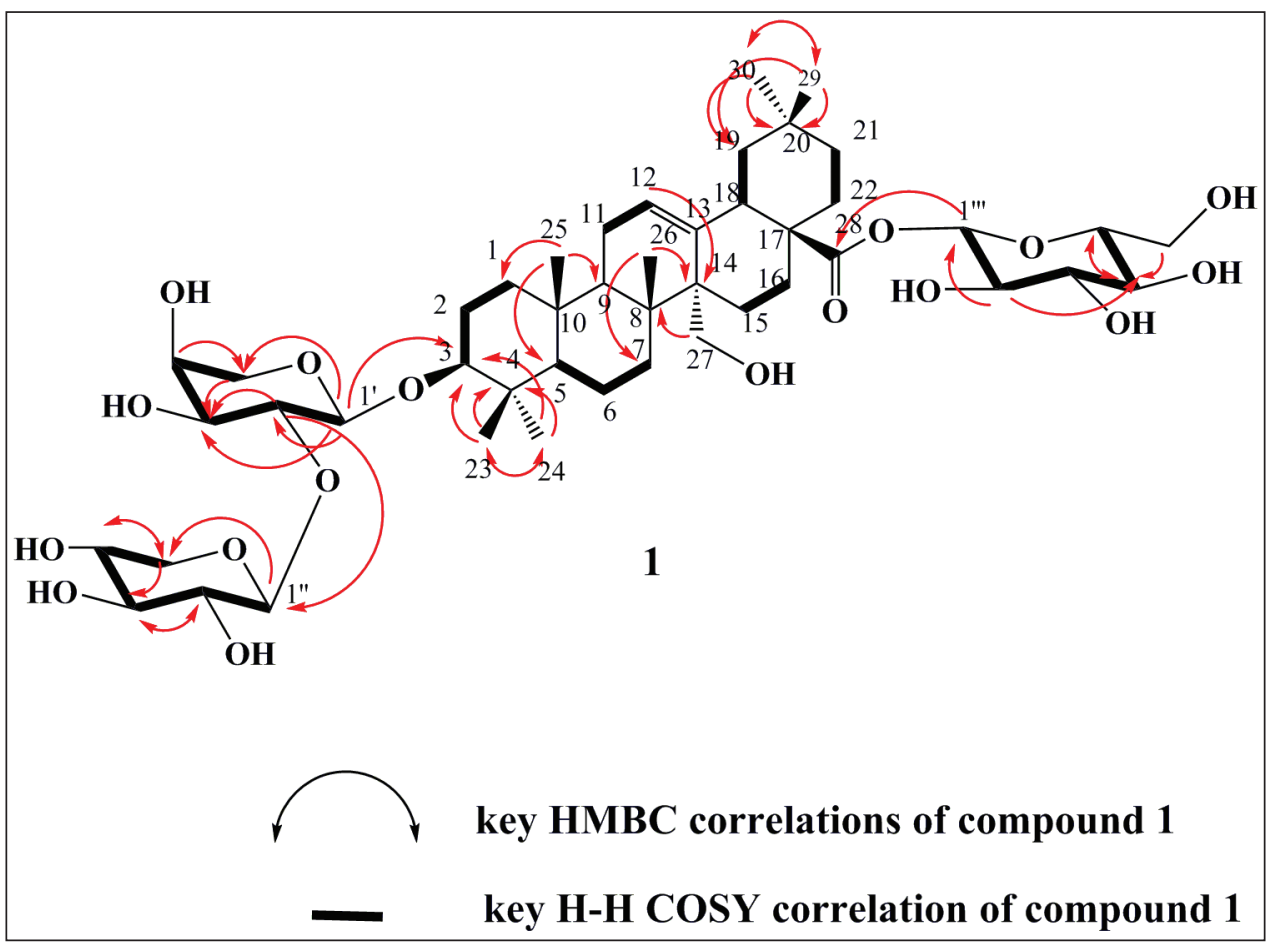

Figure 2. Key HMBC and H-H COSY correlations of compound 1. 
Table 2. Antimicrobial activity assessment of tested fractions and compound $\mathbf{1}$.

\begin{tabular}{|c|c|c|c|c|c|c|c|c|}
\hline \multirow[b]{2}{*}{ No. } & \multicolumn{2}{|c|}{ Escherichia coli } & \multicolumn{2}{|c|}{ Pseudomonas aeuroginosa } & \multicolumn{2}{|c|}{ Staphylococcus aureus } & \multicolumn{2}{|c|}{ Bacillus subtilis } \\
\hline & $\begin{array}{c}\text { Diameter of inhibition } \\
\text { zone }(\mathrm{mm})\end{array}$ & $\begin{array}{l}\text { \% Activity } \\
\text { index }\end{array}$ & $\begin{array}{c}\text { Diameter of } \\
\text { inhibition zone }(\mathrm{mm})\end{array}$ & $\begin{array}{l}\text { \% Activity } \\
\text { index }\end{array}$ & $\begin{array}{c}\text { Diameter of inhibition } \\
\text { zone }(\mathrm{mm})\end{array}$ & $\begin{array}{l}\text { \% Activity } \\
\text { index }\end{array}$ & $\begin{array}{c}\text { Diameter of } \\
\text { inhibition zone }(\mathrm{mm})\end{array}$ & $\begin{array}{l}\text { \% Activity } \\
\text { index }\end{array}$ \\
\hline 1 & 12 & 48.0 & 18 & 78.3 & 11 & 45.8 & 16 & 69.6 \\
\hline EtOAc fraction & 19 & 76.0 & 21 & 91.3 & 20 & 83.3 & 19 & 82.6 \\
\hline Crude saponin & 3 & 12.0 & 8 & 34.8 & 7 & 29.2 & 10 & 43.5 \\
\hline Ampicillin & 25 & 100 & 23 & 100 & 24 & 100 & 23 & 100 \\
\hline
\end{tabular}

EtOAc and crude saponins fractions and compound 1 were tested for their antiproliferative activity against HepG2 and MCF-7 tumor cell lines. The results illustrated that all of them inhibited the growth of HepG2 (liver cancer) and MCF-7 (breast cancer) cell lines with different sensitivity depending on cell lines. The EtOAc fraction displayed potent antiproliferative activity toward HepG2 and MCF-7 with $\mathrm{IC}_{50}$ value of $7.38 \pm 0.4$ and $4.89 \pm 0.3 \mu \mathrm{g} / \mathrm{ml}$, respectively. Additionally, compound $\mathbf{1}$ showed very potent cytotoxic activity against MCF-7 and HepG2 with $\mathrm{IC}_{50}$ value of $8.18 \pm 0.9$ and $19.20 \pm 1.4 \mu \mathrm{g} / \mathrm{ml}$, respectively. However, the crude saponins fraction showed moderate activity against HepG2 and MCF-7 with $\mathrm{IC}_{50}$ values of $42.34 \pm 3.5$ and $30.08 \pm 2.2 \mu \mathrm{g} / \mathrm{ml}$, respectively. This synergetic activity could be attributed to the presence of phenolics, flavonoids, and triterpene saponins and this was in accordance with their reported high cytotoxicity on different cell lines (Abdel-Khalik et al., 2001; Ansari et al., 1987; El-Wakil, 2007; Farheen et al., 2015; Shaker et al., 2013).

\section{Antioxidant activity}

\section{2,2,1-diphenyl-1-picrylhydrazyl method}

Shortly, DPPH, $2.7 \mathrm{ml}$ of $0.2 \mathrm{mM}$ solution, was added to the extract solution, $0.3 \mathrm{ml}$, at several concentrations. The mixture was shaken strongly and incubated for $1 \mathrm{~h}$ at room temperature before recording the absorbance at $517 \mathrm{~nm}$. The radical scavenging activity was calculated from the following equation: scavenging rate $\mathrm{D}=[(\mathrm{As}-\mathrm{Ai}) / \mathrm{As}] * 100$, where $\mathrm{As}$ is the absorbance of pure DPPH and Ai is the absorbance of DPPH in presence of extracts. Ascorbic acid in concentrations near to the experimental samples was used as reference material. DPPH antioxidant test results (antioxidant $\mathrm{IC}_{50}$ values for compound $\mathbf{1}$, EtOAc, and crude saponin fraction) are presented in Table 3. The results have revealed that EtOAc fraction was the most active one (lower $\left.\mathrm{IC}_{50}\right)$ compared to L-ascorbic acid $\left(\mathrm{IC}_{50}=26.16 \pm\right.$ $0.02 \mu \mathrm{g}$ ), while others showed relatively similar activity to that of L-ascorbic acid. The crud fractions and compound 1 showed different degrees of free radical scavenging activity toward the DPPH radical, with diminishing activity in the following order: EtOAc fraction $>$ compound $\mathbf{1}>$ crude saponins fraction, which highlights their excellent antioxidant activities.

\section{Superoxide- (SOD-) like activity}

EtOAc fraction and compound 1 exhibited strong SODlike antioxidant activity, as presented in Table 3 . The inhibition percent was $73.2 \%$ and $63.9 \%$, respectively. Crude saponins fraction showed moderate SOD-like activity, as the inhibition
Table 3. Antioxidant activity of tested fractions and compound $\mathbf{1}$ by using DPPH and SOD.

\begin{tabular}{lclcc}
\hline \multicolumn{2}{c}{ DPPH } & \multicolumn{4}{c}{ SOD } \\
\hline \multirow{2}{*}{ Compd./fraction } & Conc. $\boldsymbol{\mu} \mathbf{g} / \mathbf{m l})$ & Compd./fraction & $\begin{array}{l}\boldsymbol{\Delta} \text { through } \\
\mathbf{4} \text { minutes }\end{array}$ & \% Inhibition \\
\cline { 2 - 5 } & $\mathrm{IC}_{50}$ & Control & 0.385 & - \\
\hline Vit-C & $32.53 \pm 0.03$ & Ascorbic acid & 0.096 & 75.1 \\
1 & $33.95 \pm 0.03$ & 1 & 0.139 & 63.9 \\
Crude saponin & $52.40 \pm 0.08$ & Crude saponin & 0.225 & 41.5 \\
EtOAc fraction & $26.16 \pm 0.02$ & EtOAc fraction & 0.103 & 73.2 \\
\hline
\end{tabular}

percent was $41.5 \%$. Thus, the SOD-like activity method confirmed the same antioxidant activity pattern as follows: EtOAc fraction $>$ compound $\mathbf{1}>$ crude saponin fraction.

\section{CONCLUSION}

The chemical investigation of $F$. schimperi led to the identification of a nonpreviously described triterpene saponin (1), along with five known compounds, which are isolated here for the first time from $F$. schimperi. The EtOAc and crude saponins fractions, as well as compound 1, were evaluated for their antibacterial, antioxidant, and cytotoxic activities. The EtOAc fraction and compound 1 were found to exhibit the most potent activities

\section{CONFLICT OF INTEREST}

All the authors declare that they have no conflicts of interest for this work.

\section{FUNDING}

None.

\section{REFERENCES}

Abdel-Khalik S, Miyase T, Melek F, El-Ashaal H. Further saponins from Fagonia cretica. Die Pharm, 2001; 56:247-50.

Abdel-Khalik SA, Miyase T, El-Ashaal HA, Melek F. Triterpenoid saponins from Fagoniacretica. J Phytochem, 2000; 54:853-9.

Ansari AA, Kenne L, Atta-ur-Rahman, Wehler T. Isolation and characterization of two saponins from Fagonia indica. J Phytochem, 1987; 26:1487-90.

Boulos L. Flora of Egypt. Al Hadara Puplishing, Cairo, Egypt, 2002.

El-Demerdash A, Atanasov AG, Horbanczuk OK, Tammam MA, Abdel-Mogib M, Hooper JNA, Sekeroglu N, Al-Mourabit A, Kijjoa A. Chemical diversity and biological activities of marine sponges of the genus Suberea: a systematic review. Mar Drugs, 2019a; 17:115.

El-Demerdash A, Dawidar A, Keshk E, Abdel-Mogib M. Coumarins from Cynanchum acutum. Rev Latinoam Quím, 2009; 37:65-9. 
El-Demerdash A, Dawidar AM, Keshk EM, Abdel-Mogib M. Gingerdione from the rhizomes of Curcuma longa. Chem Nat Compd, 2012; 48:646-8.

El-Demerdash A, Genta-Jouve G, Bärenstrauch M, Kunz C, Baudouin E, Prado S. Highly oxygenated isoprenylated cyclohexanoids from the fungus Parastagonospora nodorum SN15. Phytochem, 2019b; 166:112056.

El-Demerdash A, Moriou C, Martin MT, Petek S, Debitus C, Al-Mourabit A. Unguiculins A-C: cytotoxic bis-guanidine alkaloids from the French Polynesian sponge, Monanchora n. sp. Nat Prod Res, 2018a; $32: 1512-7$.

El-Demerdash A, Moriou C, Toullec J, Besson M, Soulet S, Schmitt N, Petek S, Lecchini D, Debitus C, Al-Mourabit A. Bioactive bromotyrosine-derived alkaloids from the polynesian sponge Suberea ianthelliformis. Mar Drugs, 2018b; 16:146.

El-Rokh, AR, Negm A, El-Shamy M, El-Gindy M, AbdelMogib M. Sucrose diester of aryldihydronaphthalene-type lignans from Echium angustifolium Mill. and their antitumor activity. Phytochem, 2018; 149:155-60.

El-Wakil EA. Phytochemical and molluscicidal investigations of Fagonia arabica. Z Naturforsch C, 2007; 62:661-7.

Farheen R, Siddiqui BS, Mahmood I, Simjee SU, Majeed S. Triterpenoids and triterpenoidsaponins from the aerial parts of Fagonia indica Burm. J Phytochem Lett, 2015; 13:256-61.

Ito H, Nishitani N, Konoshima T, Takasaki M, Kozuka M, Yoshida T. Flavonoid and benzophenone glycosides from Coleogynera mosissima. J Phytochem, 2000; 54:695-700.

Kanwal N, Adhikari A, Hamed A, Hafizur RH, Musharraf SG. Isolation and characterization of non-sulfated and sulfated triterpenoid saponins from Fagonia indica. J Phytochem, 2017; 143:151-9.

Kasture VS, Gosavi SA, Kolpe JB, Deshapande SG. Phytochemical and biological evaluation of Fagonia species: a review. World J Pharm Pharm Sci, 2014; 3:1206-17.

Lam M, Carmichael AR, Griffiths HR. An aqueous extract of Fagonia cretica induces DNA damage, cell cycle arrest and apoptosis in breast cancer cells via FOXO3a and p53 expression. PLoS One, 2012; 7:e40152.

Lee Y, Jung JC, Ali Z, Khan IA, Oh S. Anti-inflammatory effect of triterpene saponins isolated from blue cohosh (Caulophyllum thalictroides). Evid Based Complement Alternat Med, 2012; 2012:798192-200.

Miyase T, Melek FR, El-Gindi OD, Abdel-Khalik SM, El-Gindi MR, Haggag MY, Hilal SH. Saponin from Fagonia arabica. J Phytochem, 1996; 41:1175-9.

Nagaraj DS, Venkateswarlu B. Antipyretic effect of aqueous extract of Fagonia cretica L. whole plant on brewer's yeast induced pyrexia in wistar rats. Int J Pharmacol Toxicol, 2013; 3:39-42.
Nazir I, Ur Rahman N, Alvi Z, Hafizur Rahman M, Sendker J, Zhang T, Frankish N, Sheridan H. Antidiabetic activities of an lc/ms finger printed aqueous extract of Fagonia cretica L. in preclinical models. Planta Med, 2017; 83:1141-8.

Perrone A, Masullo M, Bassarello C, Hamed AI, Belisario MA, Pizza C, Piacente S. Sulfated triterpene derivatives from Fagonia arabica. J Nat Prod, 2007; 70:584-8.

Prouillac C, Vicendo P, Garrigues JC, Poteau R, Rima G. Evaluation of new thiadiazoles and benzothiazoles as potential radioprotectors: free radical scavenging activity in vitro and theoretical studies (QSAR, DFT). Free Radical Biol Med, 2009; 46:1139-48.

Rahman AU, Choudhary MI, Thomsen WJ. Bioassay techniques for drug development. Harwood Academic Publishers, Amsterdam, Netherlands, p 16, 2001.

Rizk AM, Hammouda FM, Abdel-Gawad MM. The triterpenoid sapogenins of Fagonia indica Brum. J Pharm, 1972; 27:534-5.

Saleem S, Jafri L, Haq I, Chang LC, Calderwood D, Green BD, Mirza B. Plants Fagonia cretica L. and Hederanepalensis K. Koch contain natural compounds with potent dipeptidyl peptidase-4 (DPP-4) inhibitory activity. J Ethnopharm, 2014; 156:26-32.

Saleh NAM, El-Hadidi MN. An approach to chemosystematics of Zygophyllaceae. Biochem. Syst Ecol, 1977; 5:121-8.

Shaker K, Al Jubiri S, El-Hady FA, Al-Sehemi A. New compounds from Bassia muricata and Fagonia indica. Int J Pharm Sci Res, $2013 ; 23: 231-6$.

Shaker KH, Bernhardt M, Elgamal MHA, Seifert K. Sulfonated triterpenoid saponins from Fagonia indica. Z Naturforsch C, 2000; 55:520-3.

Shaker KH, Bernhardt M, Elgamal MHA, Seifert K. Triterpenoid saponins from Fagonia indica. J Phytochem, 1999; 51:1049-53.

Skehan P, Storeng R, Scudiero D, Monks A, McMahon J, Vistica D, Warren JT, Bokesch H, Kenney S, Boyd MR. New colorimetric cytotoxicity assay for anticancer-drug screening. J Natl Cancer Inst, 1990; 82:1107-12.

How to cite this article:

Ismail H, Mostafa ME, El-Demerdash A, Hanna DM, AbdelMogib M. A new triterpene saponin from Fagonia schimperi. J Appl Pharm Sci, 2020; 10(12):068-074. 